\title{
APPLICATION OF THE TWO-TEMPERATURE MODEL FOR A NUMERICAL STUDY OF MULTIPLE LASER PULSES INTERACTIONS WITH THIN METAL FILMS
}

\author{
Ewa Majchrzak ${ }^{1,2}$, Jolanta Dziatkiewicz ${ }^{1}$ \\ ${ }^{I}$ Department of Strength of Materials and Computational Mechanics \\ Silesian University of Technology, Poland \\ ${ }^{2}$ Institute of Mathematics, Czestochowa University of Technology, Poland \\ ewa.majchrzak@polsl.pl, jolanta.dziatkiewicz@polsl.pl
}

\begin{abstract}
A thin metal film irradiated by multiple laser pulses is considered. The microscale heat transfer in the domain considered is described by hyperbolic two-temperature model. This model contains two energy equations determining the heat exchange in the electron gas and the metal lattice. The problem is solved by a explicit scheme of finite difference method. The influence of separation time between two laser pulses on the electrons and lattice temperatures is discussed.
\end{abstract}

\section{Introduction}

From the mathematical point of view, exist nowadays the different models describing the microscale heat transfer [1-5], namely Cattaneo-Vernotte equation, dual phase lag model, two-temperature models and Boltzmann equation. In this paper the heat conduction in thin metal film irradiated by multiple laser pulses is described using the microscopic hyperbolic two-temperature model supplemented by boundary and initial conditions. The problem formulated is solved by means of the finite difference method. The influence of separation time between two laser pulses on the electrons and lattice temperatures is analyzed and the conclusions are formulated.

\section{Mathematical model}

The two-temperature model describes the temporal and spatial evolution of the lattice and electrons temperatures $\left(T_{l}\right.$ and $\left.T_{e}\right)$ in the irradiated metal by two coupled nonlinear differential equations $[1,2]$ (1D problem)

$$
C_{e}\left(T_{e}\right) \frac{\partial T_{e}(x, t)}{\partial t}=-\frac{\partial q_{e}(x, t)}{\partial x}-G\left(T_{e}\right)\left[T_{e}(x, t)-T_{l}(x, t)\right]+Q(x, t)
$$




$$
C_{l}\left(T_{l}\right) \frac{\partial T_{l}(x, t)}{\partial t}=-\frac{\partial q_{l}(x, t)}{\partial x}+G\left(T_{e}\right)\left[T_{e}(x, t)-T_{l}(x, t)\right]
$$

where $T_{e}(x, t), T_{l}(x, t)$ are the temperatures of electrons and lattice, respectively, $C_{e}\left(T_{e}\right), C_{l}\left(T_{l}\right)$ are the volumetric specific heats, $G\left(T_{e}\right)$ is the electron-phonon coupling factor which characterizes the energy exchange between electrons and phonons [3], $Q(x, t)$ the source function associated with the irradiation.

In a place of classical Fourier law the following formulas are introduced

$$
\begin{gathered}
q_{e}\left(x, t+\tau_{e}\right)=-\lambda_{e}\left(T_{e}, T_{l}\right) \frac{\partial T_{e}(x, t)}{\partial x} \\
q_{l}\left(x, t+\tau_{l}\right)=-\lambda_{l}\left(T_{l}\right) \frac{\partial T_{l}(x, t)}{\partial x}
\end{gathered}
$$

where $\lambda_{e}\left(T_{e}, T_{l}\right), \lambda_{l}\left(T_{l}\right)$ are the thermal conductivities of electrons and lattice, respectively, $\tau_{e}$ is the relaxation time of free electrons in metals, $\tau_{l}$ is the relaxation time in phonon collisions.

Using the Taylor expansion the equations (3) and (4) can be written in the form

$$
\begin{gathered}
q_{e}(x, t)+\tau_{e} \frac{\partial q_{e}(x, t)}{\partial t}=-\lambda_{e}\left(T_{e}, T_{l}\right) \frac{\partial T_{e}(x, t)}{\partial x} \\
q_{l}(x, t)+\tau_{l} \frac{\partial q_{l}(x, t)}{\partial t}=-\lambda_{l}\left(T_{l}\right) \frac{\partial T_{l}(x, t)}{\partial x}
\end{gathered}
$$

The laser irradiation is taken into account in the source term (c.f. equation (1))

$$
Q(x, t)=\sqrt{\frac{\beta}{\pi}} \frac{1-R}{t_{p} \delta} I_{0} \exp \left[-\frac{x}{\delta}-\beta \frac{\left(t-2 f t_{p}\right)^{2}}{t_{p}^{2}}\right]
$$

where $I_{0}$ is the laser intensity, $t_{p}$ is the characteristic time of laser pulse, $\delta$ is the optical penetration depth, $R$ is the reflectivity of the irradiated surface and $\beta=4 \ln 2$ and $f$ is the separation time between two laser pulses [6].

To define the thermal conductivity $\lambda_{e}$ and heat capacity $C_{e}$ of electrons the following formulas are widely used $[1,2,7-10]$

$$
\begin{gathered}
\lambda_{e}\left(T_{e}, T_{l}\right)=\lambda_{0} \frac{T_{e}}{T_{l}} \\
C_{e}\left(T_{e}\right)=\gamma T_{e}
\end{gathered}
$$


where $\lambda_{0}, \gamma$ are the material constants. It should be pointed out that the simple form of dependences (8), (9) is only suitable for low laser intensity. For such intensities, constant values of $C_{l}$ and $\lambda_{l}$ are also very commonly assumed.

\section{Method of solutions}

To solve the problem formulated the algorithm based on the finite difference method is proposed. A staggered grid is introduced $[8,11]$ (Fig. 1). Let us denote $T_{i}^{f}=T(i h, f \Delta t)$, where $h$ is a mesh size, $\Delta t$ is a time step, $i=0,2,4, \ldots, N, f=0$, $1,2, \ldots, F$, and $q_{j}^{f}=q(j h, f \Delta t)$, where $j=1,3, \ldots ., N-1$.

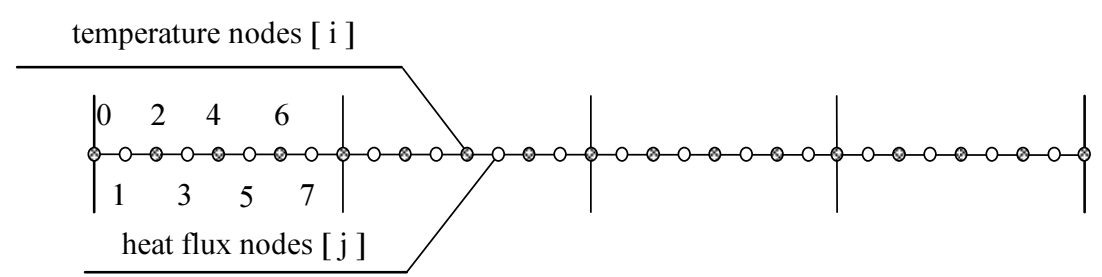

Fig. 1. Discretization

The finite difference approximation of equations (5) and (6) using the explicit scheme can be written in the form:

$$
\begin{gathered}
q_{e j}^{f-1}+\tau_{e} \frac{q_{e j}^{f}-q_{e j}^{f-1}}{\Delta t}=-\lambda_{e j}^{f-1} \frac{T_{e j+1}^{f-1}-T_{e j-1}^{f-1}}{2 h} \\
q_{l j}^{f-1}+\tau_{l} \frac{q_{l j}^{f}-q_{l j}^{f-1}}{\Delta t}=-\lambda_{l j}^{f-1} \frac{T_{l j+1}^{f-1}-T_{l j-1}^{f-1}}{2 h}
\end{gathered}
$$

where index $j$ corresponds to the 'heat flux nodes' (Fig. 1).

The dependencies (10), (11) allow one to construct the similar formulas for nodes $i-1, i+1$ and then one obtains

$$
\begin{gathered}
q_{e i-1}^{f}-q_{e i+1}^{f}=\frac{\tau_{e}-\Delta t}{\tau_{e}}\left(q_{e i-1}^{f-1}-q_{e i+1}^{f-1}\right)+ \\
\frac{\Delta t}{2 h \tau_{e}}\left[\lambda_{e i-1}^{f-1}\left(T_{e i-2}^{f-1}-T_{e i}^{f-1}\right)+\lambda_{e i+1}^{f-1}\left(T_{e i+2}^{f-1}-T_{e i}^{f-1}\right)\right] \\
q_{l i-1}^{f}-q_{l i+1}^{f}=\frac{\tau_{l}-\Delta t}{\tau_{l}}\left(q_{l i-1}^{f-1}-q_{l i+1}^{f-1}\right)+ \\
\frac{\Delta t}{2 h \tau_{l}}\left[\lambda_{l i-1}^{f-1}\left(T_{l i-2}^{f-1}-T_{l i}^{f-1}\right)+\lambda_{l i+1}^{f-1}\left(T_{l i+2}^{f-1}-T_{l i}^{f-1}\right)\right]
\end{gathered}
$$


Now, the equations (1), (2) are discretized using the explicit scheme of the finite difference method

$$
\begin{gathered}
C_{e i}^{f-1} \frac{T_{e i}^{f}-T_{e i}^{f-1}}{\Delta t}=-\frac{q_{e i+1}^{f}-q_{e i-1}^{f}}{2 h}-G\left[T_{e i}^{f-1}-T_{l i}^{f-1}\right]+Q_{i}^{f-1} \\
C_{l} \frac{T_{l i}^{f}-T_{l i}^{f-1}}{\Delta t}=-\frac{q_{l i+1}^{f}-q_{l i-1}^{f}}{2 h}+G\left[T_{e i}^{f-1}-T_{l i}^{f-1}\right]
\end{gathered}
$$

where index $i$ corresponds to the 'temperature nodes' as shown in Figure 1.

Putting (12) into (14) and (13) into (15) one has:

$$
\begin{gathered}
C_{e i}^{f-1} \frac{T_{e i}^{f}-T_{e i}^{f-1}}{\Delta t}=\frac{\left(\tau_{e}-\Delta t\right)}{2 h \tau_{e}}\left(q_{e i-1}^{f-1}-q_{e i+1}^{f-1}\right)+\frac{\Delta t}{4 h^{2} \tau_{e}}\left[\lambda_{e i-1}^{f-1}\left(T_{e i-2}^{f-1}-T_{e i}^{f-1}\right)+\right. \\
\left.\lambda_{e i+1}^{f-1}\left(T_{e i+2}^{f-1}-T_{e i}^{f-1}\right)\right]-G\left[T_{e i}^{f-1}-T_{l i}^{f-1}\right]+Q_{i}^{f-1} \\
C_{l i}^{f-1} \frac{T_{l i}^{f}-T_{l i}^{f-1}}{\Delta t}=\frac{\left(\tau_{l}-\Delta t\right)}{2 h \tau_{l}}\left(q_{l i-1}^{f-1}-q_{l i+1}^{f-1}\right)+\frac{\Delta t}{4 h^{2} \tau_{l}}\left[\lambda_{l i-1}^{f-1}\left(T_{l i-2}^{f-1}-T_{l i}^{f-1}\right)+\right. \\
\left.\lambda_{l i+1}^{f-1}\left(T_{l i+2}^{f-1}-T_{l i}^{f-1}\right)\right]+G\left[T_{e i}^{f-1}-T_{l i}^{f-1}\right]
\end{gathered}
$$

From equation (16) results that

$$
\begin{gathered}
T_{e i}^{f}=\left(1-\frac{(\Delta t)^{2} \lambda_{e i-1}^{f-1}}{4 h^{2} \tau_{e} C_{e i}^{f-1}}-\frac{(\Delta t)^{2} \lambda_{e i+1}^{f-1}}{4 h^{2} \tau_{e} C_{e i}^{f-1}}-\frac{G \Delta t}{C_{e i}^{f-1}}\right) T_{e i}^{f-1}+\frac{(\Delta t)^{2} \lambda_{e i-1}^{f-1}}{4 h^{2} \tau_{e} C_{e i}^{f-1}} T_{e i-2}^{f-1}+ \\
\frac{(\Delta t)^{2} \lambda_{e i+1}^{f-1}}{4 h^{2} \tau_{e} C_{e i}^{f-1}} T_{e i+2}^{f-1}+\frac{G \Delta t}{C_{e i}^{f-1}} T_{l i}^{f-1}+\frac{\Delta t\left(\tau_{e}-\Delta t\right)}{2 h \tau_{e} C_{e i}^{f-1}}\left(q_{e i-1}^{f-1}-q_{e i+1}^{f-1}\right)+\frac{Q_{i}^{f-1} \Delta t}{C_{e i}^{f-1}}
\end{gathered}
$$

From equation (17) results that

$$
\begin{aligned}
T_{l i}^{f}= & \left(1-\frac{(\Delta t)^{2} \lambda_{l i-1}^{f-1}}{4 h^{2} \tau_{l} C_{l}}-\frac{(\Delta t)^{2} \lambda_{l i+1}^{f-1}}{4 h^{2} \tau_{l} C_{l}}-\frac{G \Delta t}{C_{l}}\right) T_{l i}^{f-1}+\frac{(\Delta t)^{2} \lambda_{l i-1}^{f-1}}{4 h^{2} \tau_{l} C_{l}} T_{l i-2}^{f-1}+ \\
& \frac{(\Delta t)^{2} \lambda_{l i+1}^{f-1}}{4 h^{2} \tau_{l} C_{l}} T_{l i+2}^{f-1}+\frac{G \Delta t}{C_{l}} T_{e i}^{f-1}+\frac{\Delta t\left(\tau_{l}-\Delta t\right)}{2 h \tau_{l} C_{l}}\left(q_{l i-1}^{f-1}-q_{l i+1}^{f-1}\right)
\end{aligned}
$$

In numerical computations the following approximation of thermal conductivities has been used (c.f. equations (18), (19)) and (c.f. equations (10), (11)) 


$$
\begin{aligned}
& \lambda_{e i-1}^{f-1}=\frac{\lambda_{e i}^{f-1}+\lambda_{e i-2}^{f-1}}{2}, \quad \lambda_{e i+1}^{f-1}=\frac{\lambda_{e i}^{f-1}+\lambda_{e i+2}^{f-1}}{2} \\
& \lambda_{l i-1}^{f-1}=\frac{\lambda_{l i}^{f-1}+\lambda_{l i-2}^{f-1}}{2}, \quad \lambda_{l i+1}^{f-1}=\frac{\lambda_{l i}^{f-1}+\lambda_{l i+2}^{f-1}}{2} \\
& \lambda_{e j}^{f-1}=\frac{\lambda_{e j-1}^{f-1}+\lambda_{e j+1}^{f-1}}{2}, \quad \lambda_{l j}^{f-1}=\frac{\lambda_{l j-1}^{f-1}+\lambda_{l j+1}^{f-1}}{2}
\end{aligned}
$$

It should be pointed out that adequate stability criteria for explicit scheme must be fulfilled, this means (equations (10), (11))

$$
\frac{\tau_{e}-\Delta t}{2 h \tau_{e}} \geq 0, \quad \frac{\tau_{l}-\Delta t}{2 h \tau_{l}} \geq 0
$$

and (c.f. equations (18), (19)):

$$
\begin{aligned}
& \left(1-\frac{(\Delta t)^{2} \lambda_{e i-1}^{f-1}}{4 h^{2} \tau_{e} C_{e i}^{f-1}}-\frac{(\Delta t)^{2} \lambda_{e i+1}^{f-1}}{4 h^{2} \tau_{e} C_{e i}^{f-1}}-\frac{G \Delta t}{C_{e i}^{f-1}}\right) \geq 0 \\
& \left(1-\frac{(\Delta t)^{2} \lambda_{l i-1}^{f-1}}{4 h^{2} \tau_{l} C_{l}}-\frac{(\Delta t)^{2} \lambda_{l i+1}^{f-1}}{4 h^{2} \tau_{l} C_{l}}-\frac{G \Delta t}{C_{l i}^{f-1}}\right) \geq 0
\end{aligned}
$$

\section{Results of computations}

The gold film of thickness $L=100 \mathrm{~nm}\left(1 \mathrm{~nm}=10^{-9} \mathrm{~m}\right)$ is considered. Initial temperature is equal to $T_{p}=300 \mathrm{~K}$. The layer is subjected to a short-pulse laser irradiation $\left(R=0.93, I_{0}=10 \mathrm{~J} / \mathrm{m}^{2}, t_{p}=0.1 \mathrm{ps}, \delta=15.3 \mathrm{~nm}\right.$ - c.f. equation (7)). Thermophysical parameters are following: $\lambda_{l}=\lambda_{0}, \lambda_{e}=\lambda_{0} T_{e} / T_{l}$, where $\lambda_{0}=$ $=315 \mathrm{~W} /(\mathrm{mK}), C_{l}=2.5 \mathrm{MJ} /\left(\mathrm{m}^{3} \mathrm{~K}\right), C_{e}=\gamma T_{e}$, where $\gamma=62.9 \mathrm{~J} /\left(\mathrm{m}^{3} \mathrm{~K}^{2}\right), \tau_{e}=0.04 \mathrm{ps}$ $\left(1 \mathrm{ps}=10^{-12} \mathrm{~s}\right), \tau_{l}=0.8 \mathrm{ps}, G=2.6 \cdot 10^{16} \mathrm{~W} /\left(\mathrm{m}^{3} \mathrm{~K}\right)$ [2]. The problem is solved using the finite difference method under the assumption that $\Delta t=0.001 \mathrm{ps}$ and $h=1 \mathrm{~nm}$.

Figure 2 shows the comparison of numerical results for thin gold film $(x=0$ and $I_{0}=13.4 \mathrm{~J} / \mathrm{m}^{2}$ ) with experimental data presented in [2]. The line and the symbols represent calculated temperature of electrons and experimental data, respectively. The agreement of the results obtained and measured temperatures is very good.

In Figure 3 the heat source profiles for different separation times between laser pulses, namely $f=4 t_{p}, f=6 t_{p}$ and $f=8 t_{p}$, respectively are presented. 


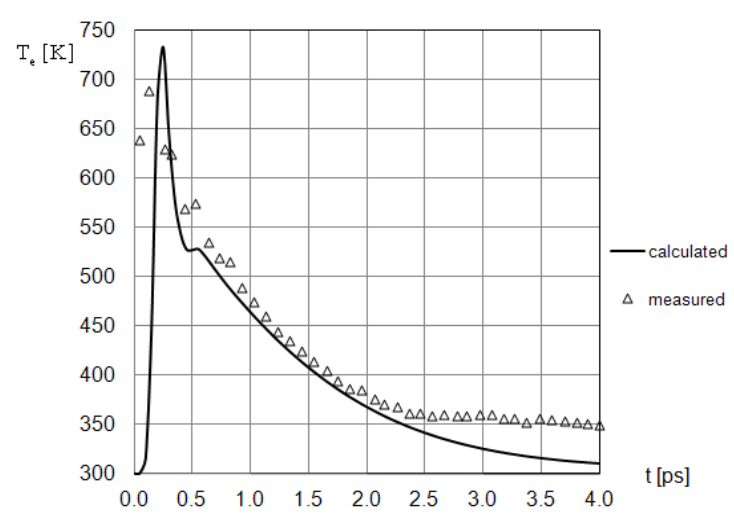

Fig. 2. Comparison of calculated electron temperature with experimental data for $100 \mathrm{~nm}$ gold film

a)

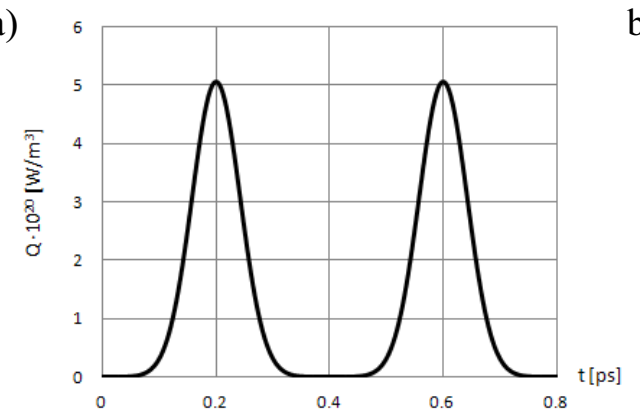

b)

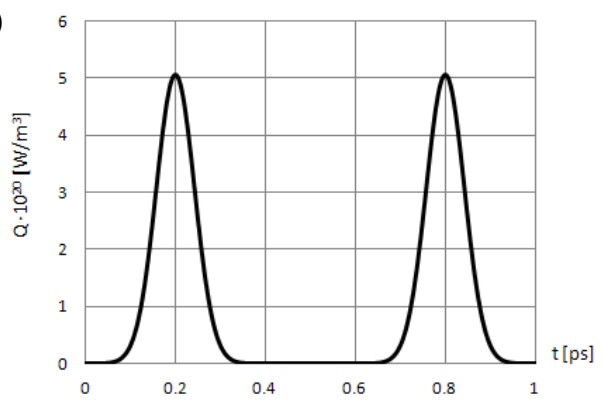

c)

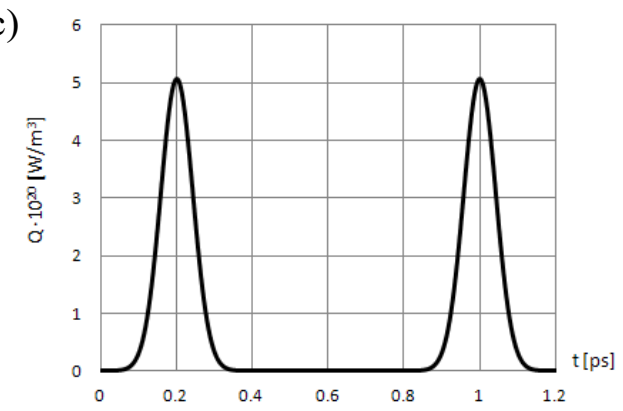

Fig. 3. Heat source profiles for different values of separation time: a) $f=4 t_{p}$, b) $f=6 t_{p}$, c) $f=8 t_{p}$

In Figures 4 and 5 the calculated electrons and lattice temperature profiles at the irradiated surface $(x=0)$ in Au thin film for different values of separation time $f$ are presented. This is visible that the separation time has a greater effect on electron temperature than the temperature of lattice. The longer separation time between laser pulses the later equilibrium of electrons and lattice temperatures is observed. 


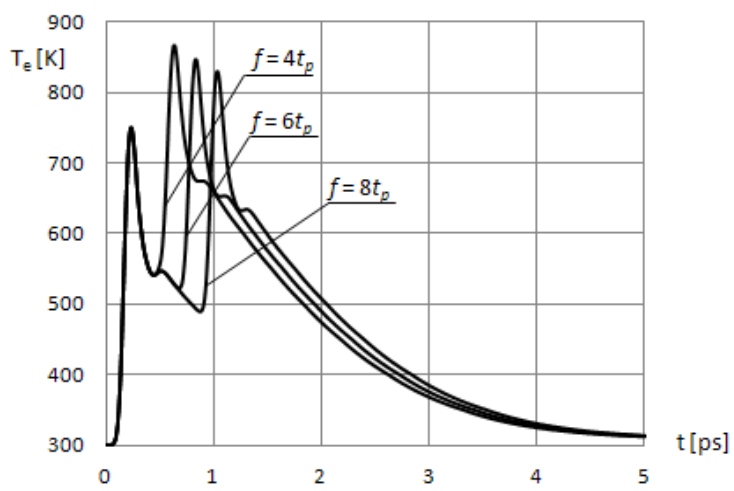

Fig. 4. Profiles of electrons temperatures for different values of $f$

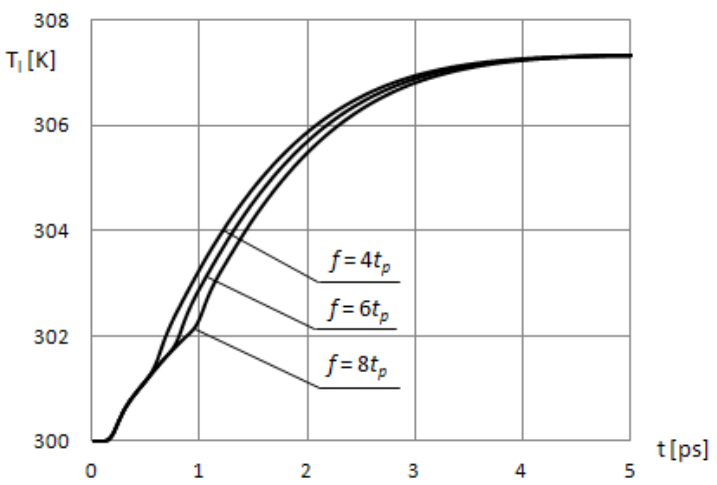

Fig. 5. Profiles of lattice temperatures for different values of $f$

\section{Conclusions}

The hyperbolic two-temperature model assures a good agreement between calculated and measured electron temperatures. It should be emphasized that the use of multiple laser pulses and different separation times allows the lattice to achieve the assumed temperature without increasing the laser power.

\section{References}

[1] Lin Z., Zhigilei L.V., Celli V., Electron-phonon coupling and electron heat capacity of metals under conditions of strong electron-phonon nonequilibrium, Physical Review B 2008, 77, 075133-1075133-17.

[2] Chen J.K., Beraun J.E., Numerical study of ultrashort laser pulse interactions with metal films, Numerical Heat Transfer 2001, Part A, 40, 1-20.

[3] Zhang Z.M., Nano/Microscale Heat Transfer, McGraw-Hill, 2007. 
[4] Mochnacki B., Paruch M., Application of evolutionary algorithms for identification of dual phase lag model parameters, Scientific Research of Institute of Mathematics and Computer Science Czestochowa University of Technology 2011, 1(10), 189-198.

[5] Mochnacki B., Paruch M., Estimation of dual phase lag model parameters using the evolutionary algorithms, Archives of Foundry Engineering 2011, 11, 3, 277-281.

[6] Huang J., Zhang Y., Chen J.K., Ultrafast solid-liquid-vapor phase change in a thin gold film irradiated by multiple femtosecond laser pulses, International Journal of Heat and Mass Transfer 2009, 52, 3091-3100.

[7] Majchrzak E., Poteralska J., Numerical modelling of short-pulse laser interactions with thin metal films using two-temperature model, $18^{\text {th }}$ International Conference on Computer Methods in Mechanics, CMM 2009, 18-21 May 2009, Zielona Góra, Short Papers, 295-296.

[8] Qiu T.Q., Tien C.L., Femtosecond laser heating of multi-layer metals - I Analysis, International Journal of Heat and Mass Transfer 1994, 37, 2789-2797.

[9] Majchrzak E., Poteralska J., Two-temperature microscale heat transfer model. Part I: Determination of electron parameters, Scientific Research of the Institute of Mathematics and Computer Science Czestochowa University of Technology 2010, 1(9), 99-108.

[10] Majchrzak E., Poteralska J., Two-temperature microscale heat transfer model. Part II: Determination of lattice parameters, Scientific Research of the Institute of Mathematics and Computer Science Czestochowa University of Technology 2010, 1(9), 109-120.

[11] Dai W., Nassar R., A compact finite difference scheme for solving a one-dimensional heat transport equation at the microscale, J. of Comput. and Applied Mathematics 2001, 132, 431-441. 